\title{
A Comparison of Routing Protocol for WSNs: Redundancy Based Approach
}

\author{
Anand Prakash \\ Invertis University, Bareilly, India \\ Email: Anand.anandprakash@gmail.com
}

\begin{abstract}
Wireless Sensor Networks (WSNs) with their dynamic applications gained a tremendous attention of researchers. Constant monitoring of critical situations attracted researchers to utilize WSNs at vast platforms. The main focus in WSNs is to enhance network localization as much as one could, for efficient and optimal utilization of resources. Different approaches based upon redundancy are proposed for optimum functionality. Localization is always related with redundancy of sensor nodes deployed at remote areas for constant and fault tolerant monitoring. In this work, we propose a comparison of classic flooding and the gossip protocol for homogenous networks which enhances stability and throughput quiet significantly.
\end{abstract}

Keywords: Wireless Sensor Network, Protocol, Message broadcasting, Classic Flooding, Gossiping flooding

\section{Introduction}

Wireless sensor network is a collection of sensor nodes organized into a cooperative network [2]. Each sensor node consists of processing capability (one or more microcontroller, CPUs or DSP chips), may contain multiple types of memory ( program, data and flash memories), have a RF transceiver (either with a omni-directional or directional antenna), have a power source (batteries or solar cells), and accommodate various sensors and actuators. The node communicate wirelessly and often self-organized after being deployed in an ad hoc fashion. WSNs are an infrastructure comprised of sensing (measuring), computing, and communication element that give an administrator the ability to instrument, observe, and react to event and phenomena in a specified environment.

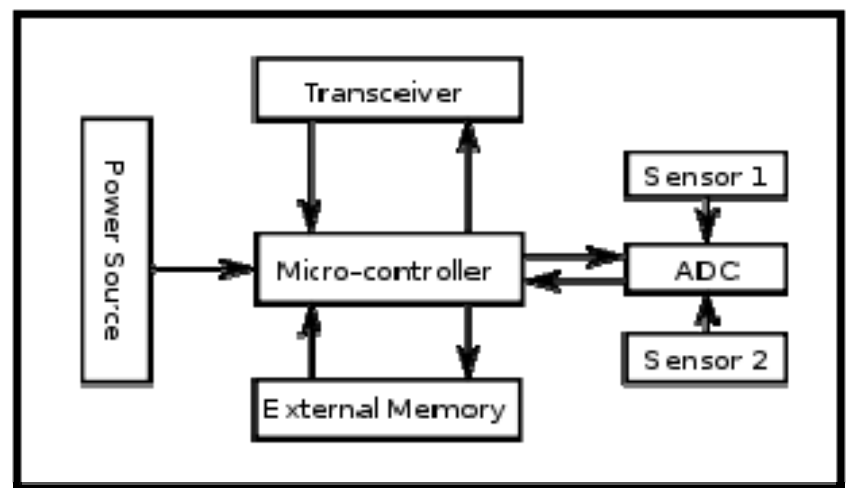

Figure 1. General model of Wireless Sensor Networks

There are four basic components in a sensor network:

1) An assembly of distributed or localized sensors.

2) An interconnecting network (usually, but not always, wireless based).

3) A central point of information clustering. 
4) A set of computing resources at the central point (or beyond) to handle data correlation, event trending, status querying, and data mining

\section{Architecture of WSNs}

Wireless sensor networks consist of individual nodes that are able to interact with the environment by sensing or controlling physical parameters. These nodes have to collaborate to full fill their tasks. The nodes are interlinked together and by using wireless links each node is able to communicate and collaborate with each other. The wireless sensor network and the classical infrastructure comprises of the standard components like sensor nodes (used as source, sink/actuators), gateways, Internet, and satellite link, etc.

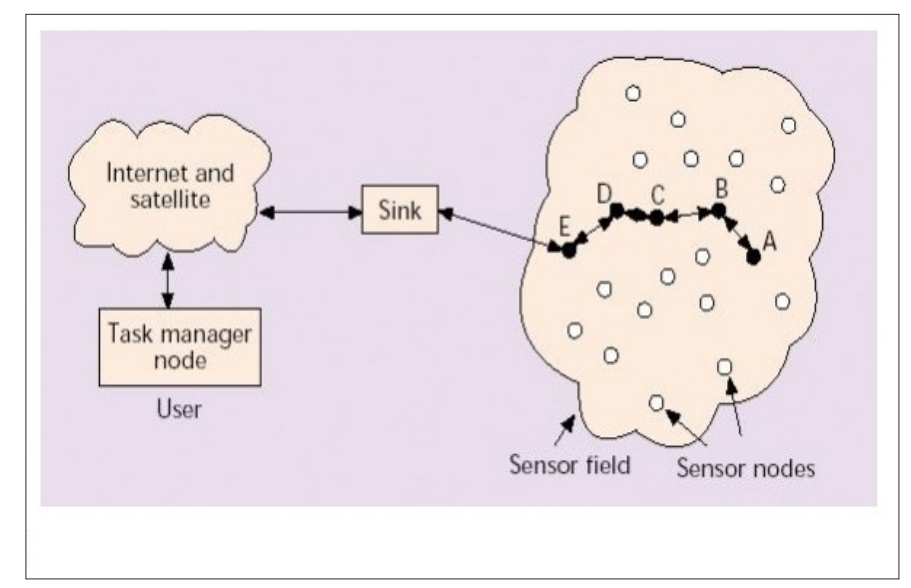

Figure 2. Sensor network communication architecture

There are many other architecture of wireless sensor network which are shown below.

\section{A. Homogeneous network}

In Homogeneous network sensor nodes or sensor field transmit data to base station. This base station forward that data over internet and from internet specific user can access the data. This type of. are classified under homogeneous network

\section{B. Heterogeneous network}

In Heterogeneous network sensor node or senor field transmit data to internet via base station and from internet the shared to different user by different means like Wi-Fi, W-Max and on any personal network. This type is classified under Heterogeneous network

\section{Ad-Hoc network}

In Ad-Hoc network the data transmit from sensor to internet and a server receive that data which is shared by $\mathrm{N}$ number of user that are wirelessly connected to server. This type is classified under Ad-Hoc network

\section{Protocol in Wireless Sensor Network}

During previous research, many differences have been observed, generally, between flat and hierarchical rout- ing protocols and, exactly, between these researched routing protocols. In this paper, we choose Gossip- ing as a target protocol to conduct our research and some extensions. Firstly a technical glimpse on Gossiping: Gossiping is a data-relay protocol, based on a Flooding protocol, and does not need routing tables or topology maintenance [10]. It was produced as an enhancement for Flooding and to overcome the drawbacks of Flooding, i.e., implosion [11]. In Flooding, a node broadcasts the data to all of its neighbors even if the receiving node has just received the same data from another node. The broadcasting will 
continue until the data is received by the destination [12]. However, in Gossiping, a node randomly chooses one of its neighbors to forward the packet to, once the selected neighbor node receives the packet it, in turn, chooses another random neighbor and forwards the packet to it. This process will continue until the destination or number of hops has been exceeded. As a result, only the selected nodes/neighbors will forward the received packet to the sink [13]. Unlike Flooding, Gossiping operates well in a one-to-one communication scenario but it does not in a one-to-many.

\section{Message Broadcasting}

Wireless sensor networks (WSNs) consist of sensor nodes that broadcast a message within a network [2]. Efficient broadcasting is a key requirement in sensor networks and has been a focal point of research over the last few years. There are many challenging tasks in the network, including redundancy control and sensor node localization that mainly depend on broadcasting. Each sensor node in the network is equipped with the memory, radio frequency transceiver, and power source to broadcast wirelessly over a specified protocol. The success of wireless sensor networks mainly depend on message forwarding/broadcasting from one node to another node [6]. And the efficiency of message broadcast depend on the protocol which are used. In this paper we compared redundancy factor of broadcasting protocol that is classic flooding protocol and gossip protocol to control redundancy and improve localization in WSNs. The proposed results incorporates the benefits of the gossip protocol for optimizing message broadcasting within the network over classic flooding.

\section{Message Broadcast Through Classic Flooding}

Classic flooding is a protocol which is used to broadcast message in WSNs. This protocol state that in a wireless sensor networks when nodes are randomly placed and the main node get message from system and distribute it to other placed node and there is no set pattern of distribution as shown in figure 3 .

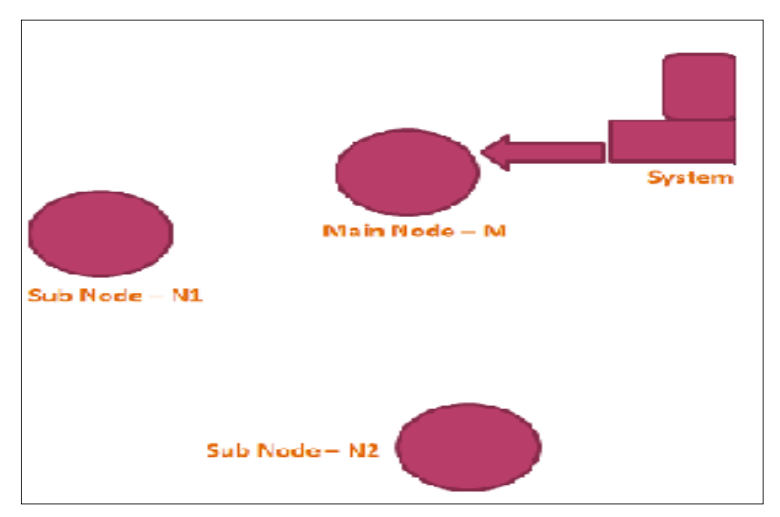

Figure 3. Classic flooding

As we can observe in figure 6 the system is transmitting data to main node (M). This main node (M) distribute it over network that is from main node (M) to sub node (N1) or from main node (M) to sub node (N2) or to both nodes (N1 \& N2). Further sub node (N1) transmit it to other nodes in network including sub node (N2) similarly sub node (N2) transmit it to other nodes in network including sub node (N1). As observed there is no set pattern of broadcasting message that is any node is transmitting to any node this leads to increase in redundancy over network. 


\section{Message Broadcast through Gossip Protocol}

Gossip protocol is a protocol which is used to broadcast message in WSNs [1]. Gossip is a probability based protocol. Whenever a sensor node wishes to send a message, it randomly selects a neighboring sensor node. On receiving the message for the first time the neighboring sensor node keep the track of messages it has already received and repeats this process to send message to neighboring node. In gossip protocol every sensor node if receive the same message twice, it is discarded. In order to achieve this, each sensor node has to keep the track of messages it has already received. Besides this the gossip protocol also performs tasks to help the inter process interaction for information exchange between networks where the sensor node failure is quite frequent as shown in figure 4.

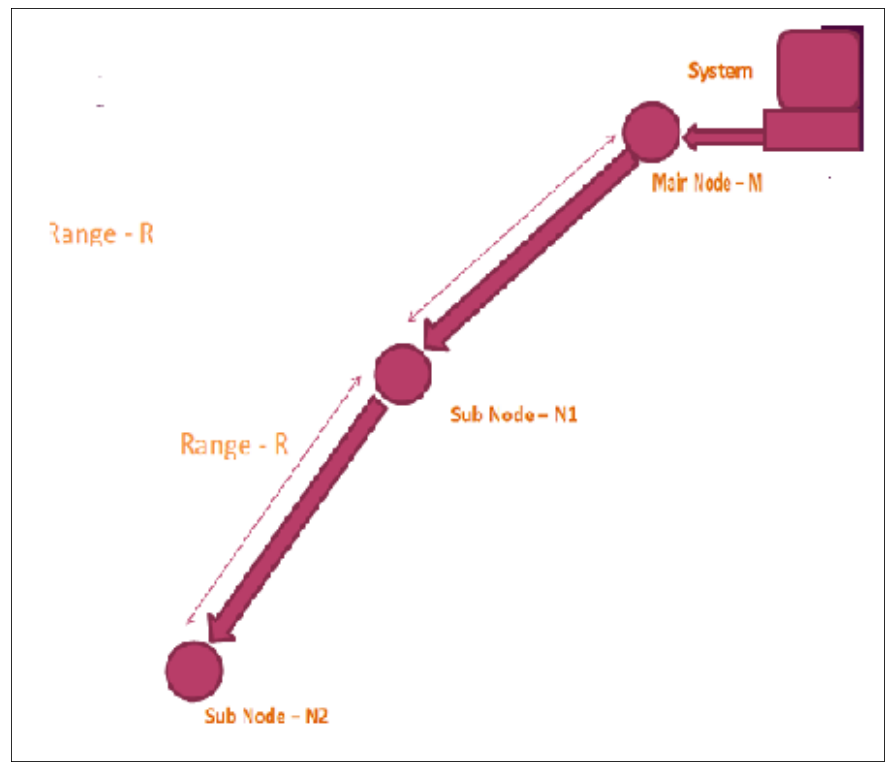

Figure 4. Gossip protocol

As we can observe that system data to main node (M) now this sensor node (M) has to keep the track of messages it received. Now this sensor node (M) randomly selects a neighboring sensor node (N1). Upon receiving the message for the first time node (N1) keep record of receive message and forward the message to other neighboring node (N2). On receiving the message for the first time the sensor node (N2) keep the track of messages it has received. If any sensor node receive the same message twice, it is discarded. Thus redundancy reduces and message is forwarded efficiently.

\section{Comparative Analysis}

We have compared the both protocol for total number of event 1 and varied total node from 10 to 200. For both protocol the result are shown below.

Classic Flooding Protocol

Case A: -Implementation of Classic flooding for total event 1 and total node 10 over width of $600 \mathrm{~m}$ and height of $500 \mathrm{~m}$ 


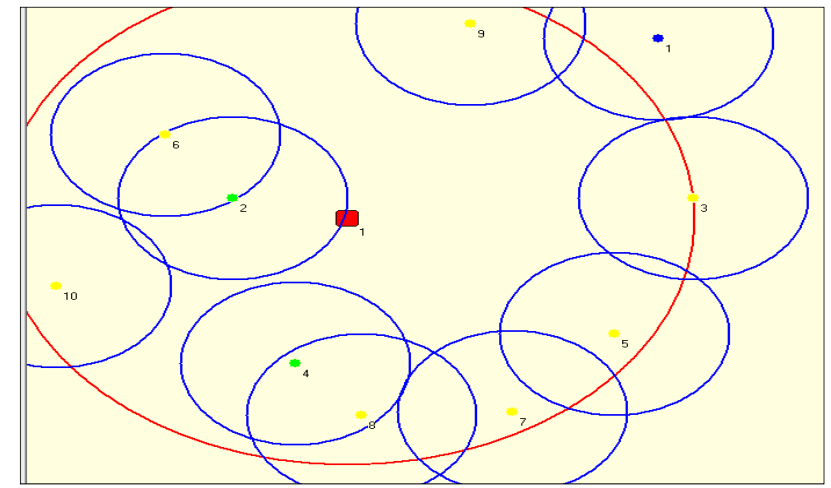

Figure 5. Classic flooding for event $=1$ and nodes $=10$

Case B: - Implementation of Classic flooding protocol for total event 1 and total node 200 over width of $600 \mathrm{~m}$ and height of $500 \mathrm{~m}$

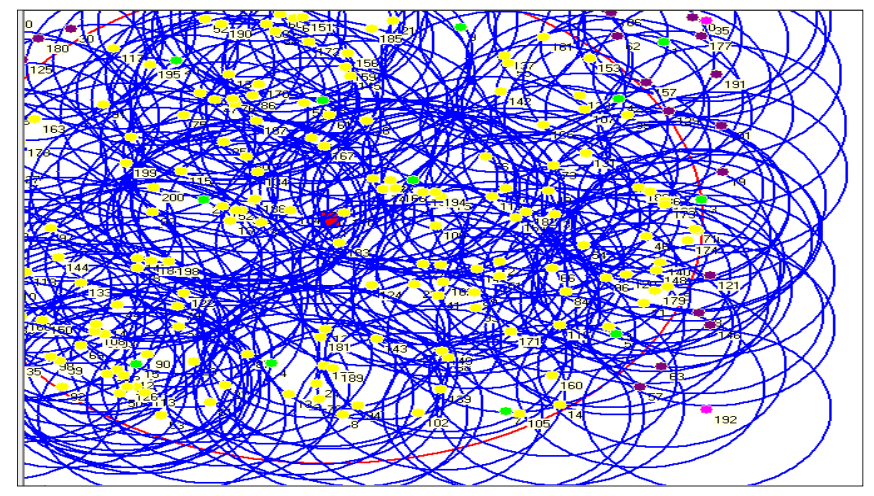

Figure 6. Classic flooding for event $=1$ and nodes $=200$

Gossip Protocol.

Case A: -Implementation of Gossip protocol for total event 1 and total node 10 over width of $600 \mathrm{~m}$ and height of $500 \mathrm{~m}$

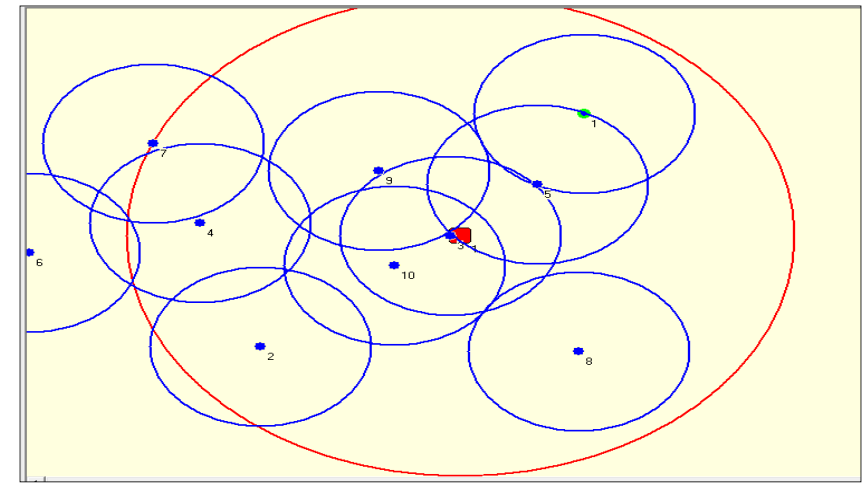

Figure 7. Gossip protocol for event $=1$ and nodes $=10$ 
Case B: -Implementation of Gossip protocol for total event 1 and total node 200 over width of $600 \mathrm{~m}$ and height of $500 \mathrm{~m}$

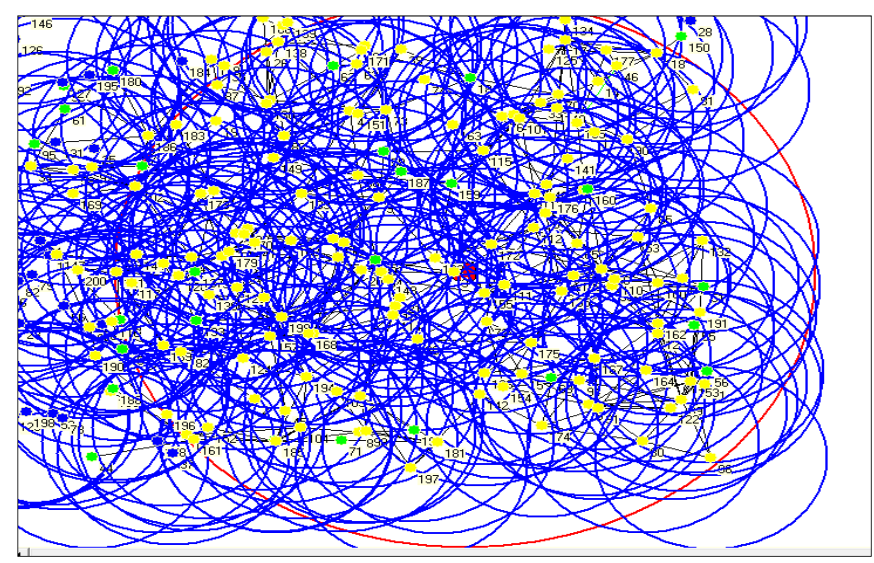

Figure 8. Gossip Protocol for event $=1$ and nodes $=10$

Graph Analysis of Classic Flooding Protocol and Gossip Protocol for number of event 1 and number of nodes 200

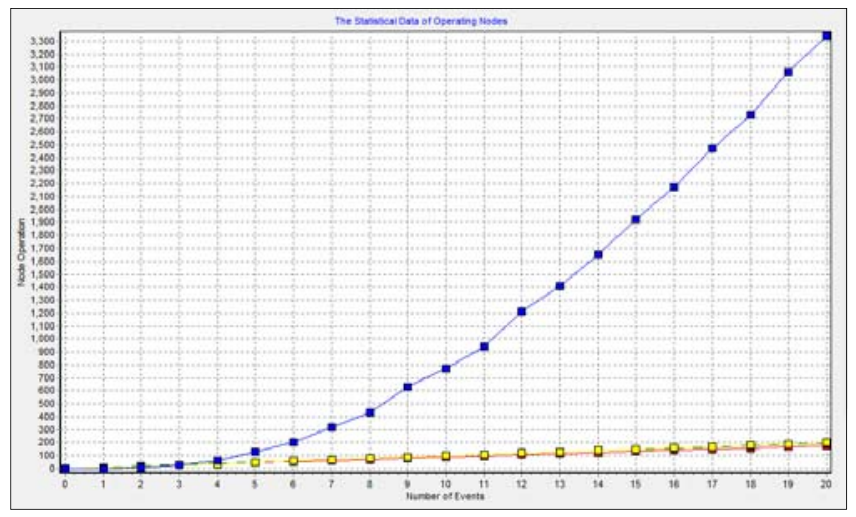

Figure 9. Classic Flooding Graph at 200 nodes

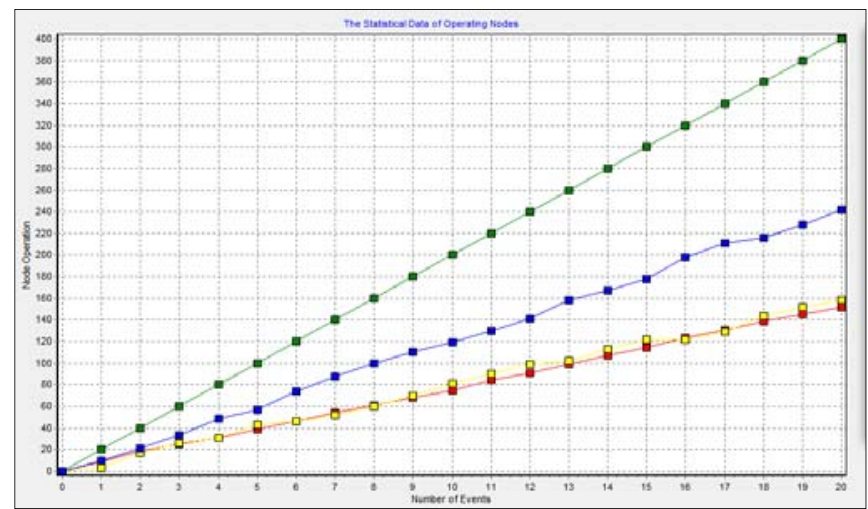

Figure 10. Gossip Protocol Graph at 200 nodes 
Analysis of redundant counts receive in both protocol with nodes varying from 10 to 200 is shown in Table 1 and Table 2.

Table 1. Classic Flooding protocol

\begin{tabular}{cccc}
\hline Sense Counts & $\begin{array}{c}\text { Transmit } \\
\text { Counts }\end{array}$ & $\begin{array}{c}\text { Receive } \\
\text { Counts }\end{array}$ & $\begin{array}{c}\text { Receive } \\
\text { Redundant } \\
\text { Counts }\end{array}$ \\
\hline 9 & 9 & 4 & 0 \\
18 & 19 & 14 & 04 \\
27 & 29 & 27 & 27 \\
35 & 38 & 37 & 63 \\
45 & 48 & 47 & 132 \\
54 & 58 & 58 & 205 \\
60 & 69 & 69 & 317 \\
70 & 79 & 79 & 435 \\
80 & 89 & 89 & 633 \\
90 & 99 & 100 & 775 \\
98 & 109 & 110 & 944 \\
108 & 119 & 120 & 1213 \\
115 & 129 & 130 & 1412 \\
124 & 139 & 140 & 1652 \\
133 & 149 & 150 & 1926 \\
142 & 159 & 160 & 2176 \\
152 & 169 & 170 & 2475 \\
159 & 179 & 180 & 2730 \\
169 & 189 & 190 & 3062 \\
177 & 199 & 200 & 3339 \\
\hline
\end{tabular}

Table 2. Gossip Protocol

\begin{tabular}{cccc}
\hline Sense Counts & $\begin{array}{c}\text { Transmit } \\
\text { Counts }\end{array}$ & $\begin{array}{c}\text { Receive } \\
\text { Counts }\end{array}$ & $\begin{array}{c}\text { Receive } \\
\text { Redundant } \\
\text { Counts }\end{array}$ \\
\hline 9 & 20 & 3 & 10 \\
19 & 40 & 17 & 21 \\
25 & 60 & 26 & 33 \\
31 & 80 & 31 & 49 \\
39 & 100 & 43 & 57 \\
46 & 120 & 46 & 74 \\
54 & 140 & 52 & 88 \\
61 & 160 & 60 & 100 \\
68 & 180 & 70 & 110 \\
75 & 200 & 81 & 119 \\
84 & 220 & 90 & 130 \\
91 & 240 & 99 & 141 \\
99 & 260 & 102 & 158 \\
107 & 280 & 113 & 167 \\
114 & 300 & 122 & 178 \\
123 & 320 & 122 & 198 \\
131 & 340 & 129 & 211 \\
139 & 360 & 144 & 216 \\
145 & 380 & 152 & 228 \\
152 & 400 & 158 & 242 \\
\hline
\end{tabular}

\section{Conclusion}

As we can observe redundant count in classic flooding protocol and Gossip Protocol are shown below in Table 3 and Table 4.

Table 3. Classic Flooding

\begin{tabular}{cccc}
\hline Protocol & $\begin{array}{c}\text { Number Of } \\
\text { Event }\end{array}$ & $\begin{array}{c}\text { Number of } \\
\text { nodes }\end{array}$ & Redundancy \\
\hline $\begin{array}{c}\text { Classic } \\
\text { Flooding }\end{array}$ & 1 & 10 & 0 \\
$\begin{array}{c}\text { Classic } \\
\text { Flooding }\end{array}$ & 1 & 200 & 3339 \\
\hline
\end{tabular}

A Comparison of Routing Protocol for WSNs: Redundancy Based Approach (Anand Prakash) 
Table 4. Gossip Protocol

\begin{tabular}{cccc}
\hline Protocol & $\begin{array}{c}\text { Number Of } \\
\text { Event }\end{array}$ & $\begin{array}{c}\text { Number of } \\
\text { nodes }\end{array}$ & Redundancy \\
\hline Gossip & 1 & 10 & 10 \\
$\begin{array}{c}\text { Protocol } \\
\text { Gossip }\end{array}$ & 1 & 200 & 242 \\
Protocol & 1 & & \\
\hline
\end{tabular}

When number of nodes are 200in network the redundant counts of classic flooding is 3339 which is very much greater that redundant counts of gossip flooding which is 242 hence gossip flooding is much better than classic flooding because redundancy factor decrease in gossip protocol hence message forwarding is more efficiently done using gossip protocol.

\section{References}

[1] AM Kermarrec, MV Steen. Gossiping In Distributed System. Operating System Review. 2007; 41(5): 2-7.

[2] IF Akyildiz, W Su, YS Sankarasubramaniam, E Cayiric. A Survey on Sensor Network. IEEE Communication Magazine. 2004; 40(8): 102-114.

[3] G Mao, B Fidan, Brian DO Anderson. Sensor Network Localization. The International Journal of Computer and Telecommunications Networking. 2007; 51: 2529-2553.

[4] Chadni and K Singh. Estimation Scheme with Improved Accuracy. International Journal of Science and Emerging Technology with Latest Trend. 2012; 3(1): 9-18.

[5] Weng Chon Ao, Kwang-Cheng Chen. Error Control for Local Broadcasting in Heterogeneous Wireless Ad Hoc Networks. IEEE Transactions on Communications. 2013; 61(4): 1510-1519.

[6] Y Liang, $\mathrm{H}$ Vincent Poor, L Ying. Secure Communication Over Wireless Broadcast Network: Stability And Utility. IEEE Transactions on Information Forensics and Security. 2011; 6(3): 682-692.

[7] Geir Egeland, Paal E Engelstad. The Economy of Redundancy in Wireless Multi-Hop Networks. In Proceeding of IEEE WCNC. 2009.

[8] Majid Khabbazian, Vijay K Bhargava. Reducing Broadcast Redundancy in WirelessAd Hoc Networks. In Proceeding of IEEE Globecom. 2007.

[9] H Modares, R Salleh, A Moravejosharieh. Overview of Security Issues in Wireless Sensor Network. In Proceeding of Third International Conference on Computational Intelligence, Modelling and Simulation IEEE. 2011: 308-311.

[10] Ian F Akyildiz, MC Vuran, B Akan, Weilian Su. Wireless Sensor Network: A Survey Revisited. Computer Networks Journal. 1-39.

[11] F Wang, J Liu. Networked Wireless Sensor Data Collection: Issues, Challenges, and Approaches. IEEE Communications Surveys \& Tutorials. 2011; 13(4); 673-687.

[12] K Islam, W Shen, X Wang. Wireless Sensor Network Reliability and Security in Factory Automation: A Survey Systems. IEEE Transactions on Systems, Man and Cybernetics, Part C: Applications and Reviews. 2012; 42(6): 1243-1256.

[13] M Erol-Kantarci, HT Mouftah, S Oktug. A Survey of Architectures and Localization Techniques for Underwater Acoustic Sensor Networks. IEEE Communications Surveys \& Tutorials. 2011; 13(3): 487502.

[14] Zhenjiang Li Yunhao Liu. Rendered Path: Range-Free Localization in Anisotropic Sensor Networks With Holes. IEEE Transactions on Networking. 2010; 18(1): 20-332.

[15] N Salman, M Ghogho, AH Kemp. Optimized Low Complexity Sensor Node Positioning in Wireless Sensor Networks. IEEE Sensors Journal. 2013; 14(1): 39-46.

[16] Chih-Yung Chang Chih-Yu Lin, Chao-Tsun Chang. Tone-Based Localization for Distinguishing Relative Locations in Wireless Sensor Networks. IEEE Sensors Journal. 2012; 12(5): 1058-1070.

[17] YW Xiaoli, G Leus. Robust Time-Based Localization for Asynchronous Networks. IEEE Transactions on Signal Processing. 2011; 59(9): 4397-4410.

IJEEI Vol. 2, No. 1, March 2014 : 48 - 55 